\title{
P01-4-8 Poster session
}

\section{Isolation of anti-allergic compound from Lotus Root}

Tomoharu Wakugawa ${ }^{1}$, Hiroyuki Mizuguchi ${ }^{2}$, Miharu Hiramatsu ${ }^{1}$, Ken-Ichi Nagamine ${ }^{3}$, Hideya Tanabe ${ }^{3}$, Keiko Shinohara ${ }^{4}$, Eiji Sawada ${ }^{4}$, Hiromichi Fujino ${ }^{1}$, Hiroyuki Fukui ${ }^{5}$, Noriaki Takeda ${ }^{6}$

${ }^{I}$ Department of Molecular Pharmacology, Institute of Biomedical Sciences, Tokushima University Graduate School, Japan, ${ }^{2}$ Laboratory of Pharmacology, Faculty of Pharmaceutical Sciences, Osaka Ohtani University, Japan, ${ }^{3}$ Nichirei Biosiences INC., Japan, ${ }^{4}$ Tokushima Agriculture, Forestry, and Fisheries Technology Support Center, Japan, ${ }^{5}$ Department of Molecular Study for Incurable Diseases, Institute of Biomedical Sciences, Tokushima University Graduate School, Japan, ${ }^{6}$ Department of Otolaryngology, Institute of Biomedical Sciences, Tokushima University Graduate School, Japan

Histamine H1 Receptor (H1R) gene is upregulated in the nasal mucosa of pollinosis patients, and its expression level is strongly correlated with severity of allergic symptoms. In nasal hypersensitivity model rats sensitized with toluene-2,4diisocyanate (TDI), long-term treatment of antihistamine suppressed TDI induced H1R mRNA up-regulation to basal level, however symptoms were not completely alleviated. These results suggested that the additional signaling is responsible for the pathogenesis of allergic symptoms. Previously we reported that suplatast tosilate inhibited nuclear factor activated T-cells (NFAT) mediated interleukin (IL)-9 mRNA expression, and combination therapy of suplatast with antihistamine markedly alleviated TDI-induced nasal symptoms. We hypothesized that suppression of NFAT mediated IL-9 mRNA upregulation could be effective for alleviating nasal symptoms in allergic rhinitis. On the other hand, traditionally Lotus Root (LR) has been used for the improvement of allergic symptoms in Tokushima, lotus-growing district in Japan. However, the underlying mechanism is unknown. We found that joint parts of LR suppressed ionomycin-induced IL-9 mRNA upregulation in RBL-2H3 cells, but it didn't suppress phorbol-12-myristate-13-acetate (PMA)-induced H1R mRNA upregulation in HeLa cells. In this study, we investigated isolation of anti-allergic compound that suppress IL-9 mRNA upregulation from LR, and effect of isolated compound on allergic symptoms in TDI-sensitized rats was evaluated. Active compounds were purified from joint parts of LR according to activity guided isolation. Isolated compound suppressed ionomycin-induced IL-9 mRNA upregulation in RBL-2H3 cells. In nasal mucosa of TDI-sensitized rats, the isolated compound also suppressed TDI-induced IL-9 gene upregulation, whereas antihistamine didn't suppress it. Combination therapy with isolated compound and antihistamine dramatically resolved allergic symptoms compared with antihistamine alone in TDI-sensitized rats. These results suggested that joint parts of LR and isolated compound resolved symptoms through the inhibition of IL-9 mRNA upregulation. Combination therapy with isolated compound and antihistamine could be effective in advanced treatment for allergic rhinitis. 